\title{
Impacto Cardiovascular da Neuropatia Autonômica do Diabetes Mellitus
}

\section{atualização}

\author{
HeLeNa SCHMid \\ Fundação Faculdade Federal \\ de Ciências Médicas de \\ Porto Alegre (FFFCMPA), \\ Universidade Federal do \\ Rio Grande do Sul (UFRGS) e \\ Santa Casa de Porto Alegre, RS.
}

As alterações neuropáticas relacionadas ao diabetes afetam o sistema nervoso somático, simpático e parassimpático. Como resultado, as complicações clínicas são extremamente variadas. Em pacientes com neuropatia autonômica ocorrem manifestações relacionadas a lesões dos sistemas genitourinário, gastrointestinal, da sudorese e cardiovascular, que, além de levarem à perda da qualidade de vida, se relacionam à morte súbita por arritmias cardíacas, bem como a aumento das taxas de mortalidade por outras causas. A neuropatia autonômica cardiovascular provavelmente contribui para o mau prognóstico da doença cardíaca coronariana e insuficiência cardíaca tanto no diabetes mellitus tipo 1 como no tipo 2. Para os diabetologistas, as complicações neurológicas do diabetes são resultado da entrada excessiva de glicose em células de tecidos como o neuronal e o endotelial. Evidências mostram que, com o objetivo de prevenir essas complicações, os pacientes diabéticos devem ser diagnosticados precocemente e instruídos a procurar um controle glicêmico adequado. O uso de inibidores da enzima conversora da angiotensina e dos bloqueadores beta-adrenérgicos é provavelmente de impacto na prevenção das complicações cardíacas do diabetes. (Arq Bras Endocrinol Metab 2007;51/2:232-243)

Descritores: Neuropatia autonômica cardiovascular; Diabetes mellitus; Doença cardiovascular

\section{ABSTRACT}

\section{Cardiovascular Impact of the Autonomic Neuropathy of Diabetes Mellitus.}

The neuropathic complications related to Diabetes may affect the somatic, sympathethic and parasympathethic nervous system. As a result, there are several clinical manifestations of diabetic neuropathy. They can be related to nervous system lesions of the genital, urinary, gastro-intestinal, skin and cardiovascular tissues. The results of these alterations are loss in the quality of life as well as increase of mortality indexes related to sudden death with cardiac arrhythmias and other causes. The cardiovascular autonomic neuropathy probably contributes to the bad prognosis of the coronary heart disease and of the heart failure in type 1 and type 2 diabetic patients. For diabetologists, the nervous complications of diabetes are the result of an increase influx of glucose to the neuronal and endothelial cells. Evidences show that, with the aim of preventing these complications, the diabetic patients should receive a precocious diagnosis and be instructed for having a good metabolic and blood pressure control. Use of angiotensin converting enzyme inhibitors and beta adrenergic blockers are probably of impact in the prevention of the cardiac autonomic complications of diabetes. (Arq Bras Endocrinol Metab 2007;51/2:232-243)

Recebido em 28/12/06 Aceito em 02/01/07
Keywords: Cardiovascular autonomic neuropathy; Diabetes mellitus; Cardiovascular disease 
$A^{s}$ NEUROPATIAS DO DIABETES (ND) estão entre as complicações crônicas mais comuns do diabetes mellitus (DM), afetando mais de $50 \%$ dos pacientes. As NDs ocorrem como conseqüência de todos os tipos de DM. As alterações neuropáticas afetam o sistema nervoso somático e o autonômico. Como resultado, as complicações clínicas das NDs são extremamente variadas, determinando importante perda da qualidade de vida dos pacientes. Em pacientes com neuropatia autonômica ocorrem manifestações relacionadas a lesões dos sistemas genitourinário, gastrointestinal, da sudorese e cardiovascular, que, além de levarem à perda da qualidade de vida, se relacionam à morte súbita por arritmias cardíacas, bem como a aumento das taxas de mortalidade por outras causas. A neuropatia autonômica cardiovascular provavelmente contribui para o mau prognóstico da doença cardíaca coronariana e insuficiência cardíaca, tanto no diabetes mellitus tipo 1 como no tipo 2 .

Para os diabetologistas, as complicações neurológicas do diabetes são resultado da entrada excessiva de glicose em células de tecidos como o neuronal e o endotelial, locais onde o transporte de glicose é controlado por transportadores que não respondem à falta de insulina absoluta ou relativa com diminuição dos níveis intracelulares de glicose. Muitos desses tecidos são suscetíveis a envelhecimento mais precoce e/ou manifestações características das complicações microvasculares em resposta a um controle metabólico nãoadequado, pressão arterial elevada e fatores genéticos. Várias evidências mostram que, com o objetivo de prevenir essas complicações, os pacientes diabéticos devem ser diagnosticados precocemente e instruídos a procurar, pelo maior tempo possível, um controle glicêmico adequado. Considerando esses aspectos, revisaremos alguns conceitos fundamentais para o entendimento da patogênese, das manifestações clínicas, dos métodos diagnósticos, da prevenção e do tratamento da neuropatia autonômica, especialmente a cardiovascular, incluindo dados que tivemos a oportunidade de obter em nossos estudos (1-13), bem como os mecanismos pelos quais essas alterações podem contribuir para aumentar o risco de pior prognóstico cardiovascular.

\section{DEFINIÇÃO DE NEUROPATIA DIABÉTICA (ND), NEUROPATIA AUTONÔMICA DO DIABETES (NAD) E NEUROPATIA AUTONÔMICA CARDIO- VASCULAR (NAC)}

As NDs têm sido definidas conforme a conferência de consenso de San Antonio: "A neuropatia diabética descreve uma desordem demonstrável, tanto clínica como subclinicamente, que ocorre na presença de diabetes mellitus sem outras causas para neuropatia periférica. As alterações neuropáticas do diabetes incluem manifestações no sistema nervoso somático e/ou no autonômico".

As neuropatias diabéticas não podem ser diagnosticadas com base num sintoma, sinal ou teste único. Um mínimo de duas anormalidades, entre sintomas, sinais, anormalidades de condução nervosa, testes quantitativos de sensibilidade ou testes autonômicos quantitativos, são necessárias $(14,15)$. O paciente é classificado como apresentando neuropatia autonômica, uma neuropatia de fibras finas, quando apresenta testes autonômicos alterados, e como apresentando neuropatia autonômica cardiovascular, quando os testes cardiovasculares estão alterados. Freqüentemente, todos os tipos de neuropatia ocorrem simultaneamente no mesmo indivíduo, variando, no entanto, o grau de comprometimento dos diferentes sistemas.

\section{CLASSIFICAÇÃO}

Várias classificações são propostas na literatura. Uma das mais utilizadas é a de Thomas (16), adaptada para Andrew Boulton (17) e apresentada na tabela 1. As

Tabela 1. Classificação das neuropatias diabéticas de acordo com Thomas $(2,3)$.

\begin{tabular}{|c|c|}
\hline Neuropatias rapidamente reversíveis & Neuropatia hiperglicêmica \\
\hline \multirow[t]{3}{*}{ Polineuropatias simétricas generalizadas } & Autonômica \\
\hline & Sensitiva aguda \\
\hline & Sensitiva motora (crônica) \\
\hline \multirow[t]{4}{*}{ Neuropatias focais e multifocais } & Cranial \\
\hline & Radiculoneuropatia toracolombar \\
\hline & Focal de um membro \\
\hline & Motora proximal (amiotrofia) \\
\hline Neuropatia inflamatória crônica & \\
\hline desmielinizante superposta & \\
\hline
\end{tabular}


diferentes formas podem ocorrer simultaneamente em um mesmo paciente. A classificação tem finalidade predominantemente didática.

A neuropatia hiperglicêmica rapidamente reversível ocorre em pacientes com diabetes recentemente diagnosticado ou transitoriamente muito descompensado. Caracteriza-se por anormalidades da condução nervosa, as quais podem estar associadas a sintomas sensitivos distais que provocam muito desconforto ao paciente. As manifestações parecem estar ligadas apenas a distúrbios funcionais, havendo remissão após restabelecimento da normoglicemia (17).

A polineuropatia generalizada simétrica é a forma mais comum de ND. Tem início insidioso relacionado à descompensação metabólica do diabetes, podendo estar presente na época do diagnóstico do diabetes mellitus tipo 2 (DM2) em mais de 10\% dos pacientes, como decorrência tanto do fato de freqüentemente o DM2 permanecer assintomático por longos períodos, como da ocorrência de neuropatia em pacientes com intolerância à glicose ainda sem critérios laboratoriais para diagnóstico de DM2 (17).

A maioria dos pacientes com neuropatia sensitivo-motora crônica apresenta-se assintomática, mas cerca de $10 \%$ podem apresentar sintomas sensitivos incapacitantes, requerendo tratamento específico. Este tipo de neuropatia é quase invariavelmente acompanhado de disfunção autonômica e de seqüelas tardias como úlceras podais, neuroartropatia de Charcot e, em muitos casos, de amputações (17).

A neuropatia sensitiva aguda é uma polineuropatia com início agudo caracterizada por muitos sintomas sensitivos e poucos achados clínicos, estando relacionada à descompensação do diabetes (17).

A neuropatia autonômica do diabetes (NAD) é também muito comum. Compromete tanto o sistema simpático como o parassimpático. Os sinais e sintomas da doença autonômica são relacionados aos sistemas cardiovascular, gastrointestinal, genitourinário, sudomotor, metabólico e oftalmológico. Estes sistemas podem mostrar evidências de comprometimento isolado ou em conjunto. Antes do desenvolvimento de testes diagnósticos mais sofisticados, o diagnóstico e a classificação da NAD eram baseados na presença de sintomas e sinais característicos, os quais, para o diagnóstico de neuropatia autonômica diabética (NAD) grave, ainda hoje são importantes, tendo-se em vista as altas sensibilidade e especificidade da presença de quatro ou mais sintomas característicos (93\% e 89\%, respectivamente) em relação à presença de NAD diagnosticada por testes cardiovasculares não-invasivos (1). Geralmente os sintomas aparecem insidiosamente, podendo, em fases iniciais, passar desapercebidos se não cuidadosamente questionados (18).

Entre as apresentações clínicas mais comuns, as que envolvem o sistema cardiovascular são taquicardia de repouso, intolerância ao exercício, arritmias cardíacas e hipotensão postural. Quando o sistema gastrointestinal é afetado, pode-se observar pirose, disfagia, odinofagia, náuseas, vômitos, plenitude gástrica, diarréia, constipação e incontinência fecal. Os distúrbios genitourinários incluem bexiga neurogênica, infecções urinárias de repetição, disfunção sexual masculina (impotência sexual e ejaculação retrógrada) e feminina (diminuição de libido e de lubrificação vaginal). Outras manifestações envolvem anormalidades pupilares (menor diâmetro da pupila em resposta ao escuro e pupila do tipo Argyll-Robertson), áreas de anidrose simétrica e sudorese gustatória paradoxal em face e parte superior do tórax em resposta a certos alimentos, e, do ponto de vista metabólico, a ocorrência de hipoglicemias assintomáticas e diminuição da resposta dos hormônios contra-reguladores às hipoglicemias $(18,19)$.

\section{PREVALÊNCIA DA NEUROPATIA AUTONÔMICA CARDIOVASCULAR (NAC)}

A prevalência relatada de NAC varia com a população estudada e métodos utilizados. Independentemente desta variação, NAC parece ser freqüente entre pessoas com diabetes. Em uma revisão de 15 artigos, a prevalência sugerida vai de $2,6 \%$ a $90 \%$ entre os diabéticos, com uma prevalência média de 30\% (18).

Inicialmente descrita como uma complicação tardia do diabetes, disfunção autonômica cardiovascular subclínica pode ser detectada mesmo em pacientes com DMl recentemente diagnosticado (20).

A prevalência de disfunção erétil completa, ajustada para idade, é de $28 \%$ em homens com DM tratado, em estudo realizado em Massachusetts. Considerando-se vários estudos, encontra-se disfunção erétil em 20-52\% dos pacientes com DMl e em 36-54\% dos portadores de DM2. Entre os fatores de risco da impotência no diabetes está a presença de neuropatia autonômica cardiovascular (20).

\section{PATOGÊNESE DAS NEUROPATIAS DIABÉTICAS}

Vários mecanismos são propostos, todos embasados em estudos experimentais e clínicos, de modo que, mais provavelmente, assim como ocorre para as outras complicações do diabetes mellitus, vários fatores pato- 
genéticos estejam envolvidos, tanto no início como na progressão da doença.

Didaticamente podemos dividir a etiologia das neuropatias diabéticas como de causas metabólica e vascular, as quais interagem entre si. Um esquema dos passos envolvidos em resposta ao aumento do fluxo de glicose para os neurônios está apresentado na figura 1.

Os resultados do DCCT (1993) sugerem que o fator isolado mais importante no desenvolvimento das complicações crônicas do diabetes mellitus é a hiperglicemia (21). O aumento dos níveis de glicose no nervo e células endoteliais determina alterações nos níveis de lípides, polióis, além de glicação protéica não-enzimática e disfunção em fatores de crescimento $(22,23)$.

Em vários tecidos não insulino-sensíveis, a glicose é metabolisada pela via dos polióis em sorbitol e frutose, respectivamente pela ação das enzimas aldose redutase e desidrogenase do sorbitol. No diabetes mellitus, como resultado da hiperglicemia, a via dos polióis é ativada tanto em neurônios como em células endoteliais, o que leva ao acúmulo de sorbitol intracelular com diminuição compensatória de mioinositol. A queda dos níveis de mioinositol associa-se à redução na síntese e turnover de fosfoinositol. A depleção de mioinositol em neurônios de ratos diabéticos associa-se à menor atividade da Na-K-ATPase e diminuição da velocidade de condução nervosa, e suplementação de mioinositol na dieta ou o uso de inibidores da aldose redutase revertem estas alterações (24).
Redução no fluxo sangüíneo nervoso, aumento de resistência vascular e menor tensão de oxigênio em nervos ciáticos de ratos diabéticos também têm sido descritos (24). Biópsias de nervos surais de humanos demonstraram alterações vasculares como espessamento de membrana basal, edema e proliferação de células endoteliais, agregação plaquetária e oclusões de vasos (25). Esses dados sugerem que, pelo menos em parte, a perda de fibras mielinizadas característica do diabetes mellitus deve-se a dano secundário à isquemia e hipóxia (25-27).

Uma unificação das hipóteses metabólica e vascular é sugerida. Vários autores acreditam que precocemente, no início do diabetes mellitus, defeitos metabólicos levam à ativação da via da aldose redutase e sorbitol desidrogenase. O óxido nítrico é um radical altamente reativo, mediador de vasodilatação e neurotransmissor inibitório, sintetizado pela sintetase do óxido nítrico, que é dependente do fosfato de nicotinamida adenina dinucleotídeo (NADPH). O fluxo de glicose pela via dos polióis diminui a disponibilidade de NADPH, o que limita reações dependentes da glutationa e também a atividade da sintase do óxido nítrico, levando à sua depleção. A depleção de óxido nítrico leva a alterações de fluxo sangüíneo no nervo, o que, por sua vez, diminui a capacidade do nervo em tamponar radicais livres, além de esgotar as reservas energéticas disponíveis. Essa falha energética promoveria o início da neuropatia diabética. $\mathrm{O}$ uso de inibidores da aldose-redutase, bloqueando a formação de

\section{Stress Oxidativo e Neuropatia}

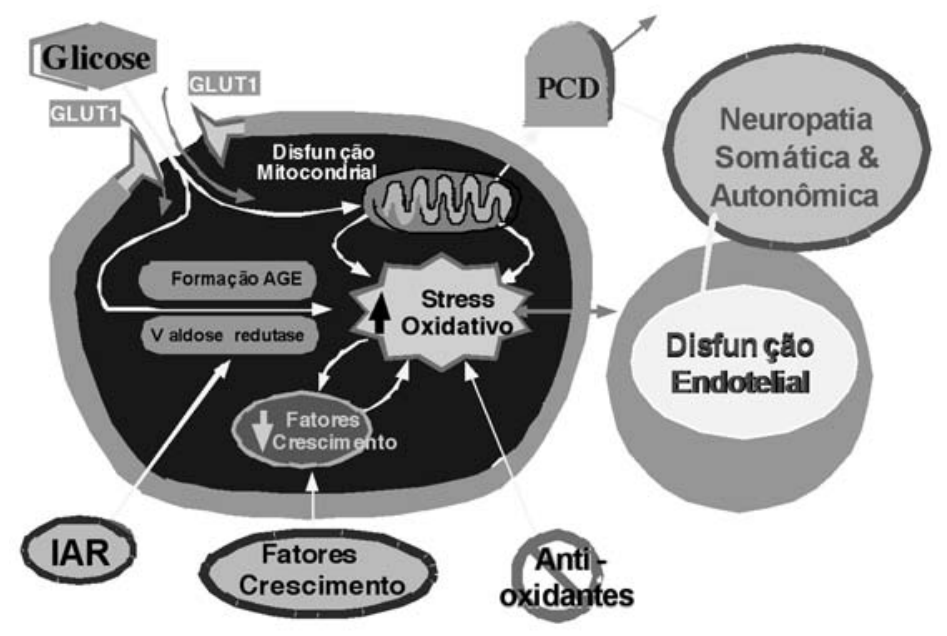

Figura 1. Esquema teórico sobre os mecanismos do aumento do estresse oxidativo e suas repercussões na patogênese da polineuropatia diabética.

V - via; PCD - morte celular programada; AGE - produtos finais de glicação avançada; IAR inibidores da aldose redutase; GLUT1 - transportador de glicose 1. 
sorbitol e, portanto, impedindo a depleção de NADPH, preveniu o aparecimento de neuropatia diabética em ratos com diabetes por estreptozotocina. A associação destes com o inibidor da sintetase do óxido nítrico (L-NAME) permitiu novamente que os animais desenvolvessem neuropatia diabética, dados que reforçam as teorias de que fluxo contínuo de glicose através da via dos polióis e aumento do estresse oxidativo (28) limitam a síntese de óxido nítrico por causar depleção de NADPH, e a diminuição de óxido nítrico resulta em vasoconstrição, isquemia e diminuição da velocidade de condução nervosa (26).

Também tem sido sugerido que uma deficiência do fator de crescimento do nervo esteja implicada na patogênese da neuropatia diabética (29). Em ratos com diabetes por estreptozotocina de longa duração, observou-se correlação das alterações no fator de crescimento do nervo com as observadas na inervação miocárdica, avaliada através de métodos radioisotópicos (2).

Em ratos normais desmamados e alimentados com uma dieta deficiente em alfatocoferol, foi observada peroxidação lipídica endoneural, que se associou ao desenvolvimento de neuropatia sensitiva (30), o que sugere que o estresse oxidativo em um rato normal produz sinais de neuropatia. A queda do estresse oxidativo poderia ser a principal função dos inibidores da aldose-redutase, assim como a elevação da atividade da glutationa (principal alvo mitocondrial para a detoxificação de peróxido de hidrogênio) e o aumento de radicais de oxigênio reativos (24).

Os possíveis mecanismos fisiopatogênicos, bem como as inter-relações entre eles, foram reunidos em esquema apresentado (figura 1 ). Nele fica evidente a natureza multifatorial dos danos, o que sugere a necessidade de múltiplas ações terapêuticas para reversão $(24,26,31)$. Além disso, é importante considerar que a deficiência de fator de crescimento do nervo seja mais importante no aparecimento da neuropatia autonômica, devido ao seu papel importante na regeneração de fibras finas.

\section{DIAGNÓSTICO DA NAC}

A NAC está associada com taquicardia de repouso, intolerância ao exercício, hipotensão ortostática e isquemia miocárdica silenciosa.

Freqüentemente o diagnóstico de neuropatia autonômica passa a ser pesquisado quando o paciente apresenta sintomas relacionados à desnervação autonômica, tais como hipotensão postural, taquicardia de repouso, intolerância ao exercício, isquemia miocárdica ou infarto sem dor. Os testes diagnósticos, no entanto, freqüentemente se alteram muito antes do aparecimento destes sintomas. O risco de neuropatia autonômica aumenta com a duração do diabetes e grau de controle glicêmico e tende a ocorrer paralelamente com o desenvolvimento de outras doenças de órgãos-alvo relacionadas ao diabetes, tais como a retinopatia, nefropatia e vasculopatia. Sintomas e sinais de desnervação autonômica freqüentemente ocorrem tardiamente na história natural desta complicação, de modo que a neuropatia autonômica poderia determinar repercussões sobre o prognóstico cardiovascular e não estar sendo reconhecida. Muitos diabetólogos entendem a neuropatia autonômica cardiovascular como uma complicação importante do diabetes mellitus tipo 1 , devido ao desafio que representa manejar essa complicação nesses pacientes e pelo fato de ela freqüentemente dominar o quadro clínico e necessidades terapêuticas desses pacientes. A NAC tende a ser menos plenamente expressa em pacientes com DM2, os quais são tipicamente mais idosos e têm grande variedade de co-morbidades concomitantemente.

\section{Métodos de avaliação da desnervação autonômica cardiovascular}

\section{Métodos não-invasivos}

Através desses testes, o diagnóstico da neuropatia autonômica pode preceder o aparecimento de sintomas. Os testes, desenvolvidos inicialmente por Ewing e cols. na década de 1970, baseiam-se em variações da freqüência cardíaca e da pressão arterial sob estímulos fisiológicos como respiração profunda, manobra de Valsalva e mudança de decúbito (18).

Os testes clássicos propostos por Ewing são cinco, baseados nos reflexos cardiovasculares e propostos como forma de diagnóstico e seguimento da neuropatia autonômica diabética (32). São eles: as respostas da freqüência cardíaca às manobras de Valsalva, levantar e respiração profunda, e as respostas da pressão arterial ao ortostatismo e à contração isométrica sustentada (32). Os autores mostraram que a existência de anormalidades nos reflexos cardiovasculares espelhava dano de todo o sistema nervoso autonômico, tendo em vista a correlação entre os testes propostos e a presença de disfunção autonômica em outros órgãos, como sistema gastrointestinal $(32,33)$, pupila etc. (34). Além disso, os testes apresentam valor prognóstico, já que a presença de sintomas associados a testes cardiovasculares alterados foi preditiva de taxa elevada de mortalidade $(56 \%) \mathrm{em}$ cinco anos (35-38). 
A Associação Americana de Diabetes (Consensus Statement, 1992) tem proposto que pelo menos três testes cardiovasculares sejam utilizados para o diagnóstico de neuropatia autonômica (por exemplo, variação do intervalo RR do eletrocardiograma durante a respiração profunda e manobra de Valsalva e variação de pressão na posição supina). Esses testes devem ser padronizados e realizados nas mesmas condições, uma vez que as respostas variam conforme horário, condição metabólica, uso de café, insulina ou tabaco, drogas de efeito cardiovascular etc. (15).

A taxa de freqüência cardíaca, monitorizada eletrocardiograficamente, é avaliada após respiração profunda, manobra de Valsalva e ortostatismo. A resposta da pressão arterial à força manual sustentada ou ao ortostatismo é medida com o esfigmomanômetro aneróide. Uma descrição pormenorizada desses testes pode ser obtida em estudos publicados previamente pelo nosso grupo $(3,4)$. Os pacientes são classificados como portadores de neuropatia autonômica na presença de dois ou mais testes alterados.

Já na época em que foram descritos, observouse que esses tipos de avaliação poderiam não diagnosticar tão precocemente a NAD, já que alguns pacientes com sintomas sabidamente causados por ela apresentavam testes normais (4). No entanto, os testes propostos por Ewing ainda hoje são, em geral, a primeira escolha na investigação da disfunção autonômica do diabetes mellitus, posto que foram padronizados e existem vários estudos em longo prazo demonstrando seu valor prognóstico, o que não ocorre em relação às novas alternativas diagnósticas (18).

Na tentativa de obter testes da função autonômica mais sensíveis, e que avaliassem as respostas do sistema nervoso autonômico a estímulos fisiológicos, Ewing e cols., em 1983 e 1984, descreveram novos métodos utilizando monitorização eletrocardiográfica de $24 \mathrm{~h}$, métodos que avaliam o ritmo circadiano da freqüência cardíaca e da pressão arterial (análise espectral). A vantagem destes testes também reside no fato de que menor participação ativa dos pacientes é necessária. Pacientes portadores de diabetes mellitus com neuropatia autonômica apresentaram perda progressiva do padrão da freqüência cardíaca de $24 \mathrm{~h}$ normal, seguindo-se piora do quadro autonômico, com perda da variação da freqüêencia cardíaca diurna normal, e maior freqüência cardíaca ao acordar e durante o sono. Além disso, cerca de $50 \%$ dos pacientes com testes clássicos inalterados demonstraram ritmo circadiano da freqüência cardíaca alterado, o que sugere que este teste é mais sensível na detecção de dano parassimpático precoce $(39,40)$.
Mais recentemente, o uso da servopletismomanometria (Finapres) permitiu avaliar, de forma nãoinvasiva, respostas da freqüência cardíaca a manobras de Valsalva, contração isométrica sustentada e mudanças posturais. O uso da servopletismomanometria (Finapres) também permite avaliar, de forma nãoinvasiva, respostas da pressão arterial a manobras que alteram seu controle (4l).

\section{Métodos que avaliam a inervação simpática cardíaca através de radioisótopos}

Métodos que utilizam radioisótopos captados especificamente por fibras adrenérgicas também têm sido utilizados para avaliar a ocorrência de neuropatia autonômica. Análogos radiomarcados da norepinefrina, que são retidos pelos terminais nervosos simpáticos do coração, permitem o mapeamento da integridade simpática cardíaca $(33,42)$. Entre eles destacamse os que utilizam a meta-iodo-benzilguanidina 123I (123I MIBG) ou a 11C-hidroxiefedrina (11C-HE), sendo visualizados por técnicas cintilográficas ou por tomografia por emissão de pósitrons (PET).

A meta-iodo-benzilguanidina, quando marcada com 123I, permite a detecção de nervos simpáticos que captam esta substância, o que ocorre em órgãos ricamente inervados, como o coração. Sua utilização no estudo da inervação simpática cardíaca baseia-se no fato de que, após a fase de captação, seu efluxo do tecido extraneuronal é maior do que o do tecido neuronal (42). Redução da captação miocárdica de 123I MIBG em diabéticos com testes cardiovasculares clássicos alterados, e também com resultados alterados de análise espectral da freqüência cardíaca, foi observada por alguns autores, além da constatação de que alguns diabéticos com testes normais podem ter alteração na captação daquele radioisótopo. Estes resultados sugerem que a cintilografia miocárdica com 123I MIBG é método não-invasivo útil no estudo da disfunção simpática cardíaca e provavelmente mais sensível do que os métodos clássicos de diagnóstico da NAD (43-46).

$\mathrm{O}$ radiotraçador $1 \mathrm{lC}-\mathrm{HE}$, recentemente desenvolvido como análogo da norepinefrina para PET, é especificamente captado e retido nos terminais simpáticos nervosos, o que permite caracterização regional quantitativa da disfunção e da perda de neurônios simpáticos (47). Anormalidades na retenção cardíaca de 11C-HE podem ser detectadas em $40 \%$ dos diabéticos com testes autonômicos clássicos normais. Indivíduos com NAD leve apresentam defeitos de captação apenas na parede inferior distal do ventrículo esquerdo, enquanto que os neuropatas graves têm envolvimento também de paredes ântero-laterais e inferiores. Além 
disso, esses pacientes apresentam cerca de 33\% de aumento na captação de $1 \mathrm{lC}-\mathrm{HE}$ nos segmentos miocárdicos proximais, sugerindo hiperinervação simpática nesta região $(48,49)$ (figuras 2 e 3 ).

\section{PROGNÓSTICO DA NAC E MORTALIDADE}

A NAC tem sido apontada como uma das possíveis causas de morte súbita. Estudos longitudinais acompanhando diabéticos neuropatas demonstraram que a mortalidade destes pacientes é de $16 \%$ a $53 \%$ em cinco anos, as maiores taxas de mortalidade ocorrendo na presença de NAD avançada. Maser e cols. (18) revisaram 15 estudos que envolveram 2.900 pacientes diabéticos com NAC. Durante o seguimento, que variou de 0,5 a 16 anos, a mortalidade foi consistentemente maior entre os pacientes com NAC (30\%) vs. a mortalidade dos que não apresentavam NAC no período basal (13\%). Reunidos os dados, foi estimado que a razão de chances para ter NAC nestes estudos foi de 3,45 (IC 2,66 a 4,47; p< 0,001) para estudos usando 2 ou mais indicadores de NAC e 1,2 (IC 1,02 a 1,41; $\mathrm{p}<0,03$ ) para estudos que utilizaram apenas uma única medida para definir a NAC.

O comprometimento autonômico pode estar evidente em vários órgãos e sistemas, como cardiovascular, gastrointestinal, pupilar e urogenital. No sistema cardiovascular, a perda do controle simpático pode levar à hipotensão postural, que se manifesta por ton-

\section{DM1 com microalbuminúria - Desnervação Simpática Leve}

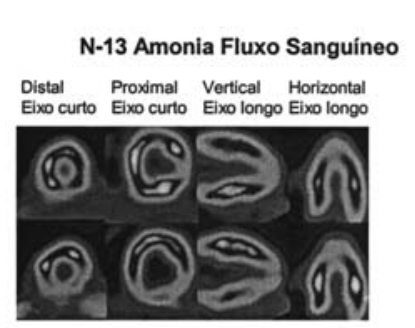

C-11 HED

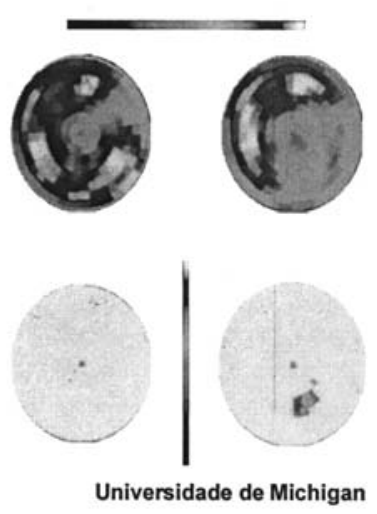

Figura 2. Mapeamento cardíaco (PET SCAN), utilizando como radiotraçadores a amônia- $\mathrm{N}-13$, para avaliação do fluxo sangüíneo, e a hidroxiefedrina-C11, para avaliação da inervação simpática. No exemplo, paciente com diabetes mellitus tipo 1, com microangiopatia e desnervação simpática leve. turas, distúrbios visuais ou síncope. Essas queixas podem ser encontradas em até 30\% dos pacientes com NAC (18).

Em resposta ao exercício, indivíduos com NAC apresentam menor aumento de freqüência cardíaca e pressão arterial, associado a menor débito cardíaco. Devido à possibilidade de intolerância a exercício, recomenda-se que indivíduos que possam ter NAC sejam submetidos a testes de estresse para avaliação de cardiopatia isquêmica antes de iniciar um programa de exercícios (18).

A hipotensão ortostática é definida como queda de $20 \mathrm{mmHg}$ na pressão arterial sistólica (PAS) ou 10 $\mathrm{mmHg}$ na diastólica (PAD) um minuto após o paciente mudar a posição de supina para ortostática. Em pacientes com diabetes, geralmente a hipotensão ortostática é o efeito do dano das fibras vasomotoras aferentes simpáticas que inervam os vasos esplâncnicos. Muitos pacientes com hipotensão postural apresentam, ao mudar da posição supina para a sentada ou de pé, tonturas, fraqueza, borramento visual e dor cervical, entretanto muitos outros permanecem assintomáticos (18).

Uma associação entre isquemia miocárdica silenciosa e NAC vem sendo mostrada em vários estudos. Nestes, a percepção da angina estava geralmente comprometida nos pacientes diabéticos, permitindo a estes indivíduos se exercitar por mais tempo após o início da isquemia miocárdica. Nesses estudos, os investigadores sugerem que um dano às fibras aferentes sen-

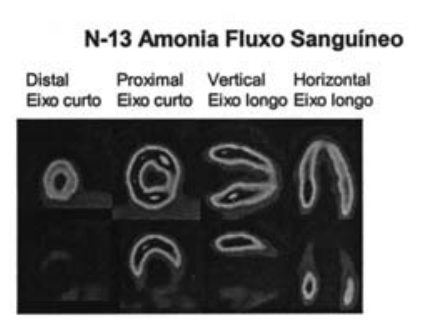

C-11 HED
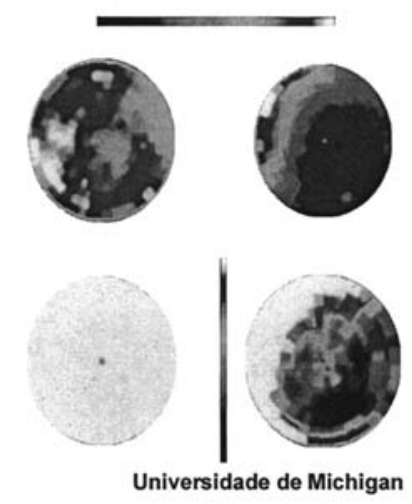

Figura 3. Mapeamento cardíaco (PET SCAN), utilizando como radiotraçadores a amônia-N-13, para avaliação do fluxo sangüíneo, e a hidroxiefedrina-C11, para avaliação da inervação simpática. No exemplo, paciente com diabetes mellitus tipo 1, com microangiopatia e desnervação simpática grave. 
sitivas ao suprimento nervoso autonômico diminui a sensibilidade de diabéticos à isquemia regional por interromper a transmissão do estímulo (18).

A presença de NAC, por outro lado, não exclui infarto do miocárdio doloroso entre indivíduos diabéticos. Dor torácica de qualquer localização em paciente com diabetes mellitus deve ser considerada de origem miocárdica até que se prove o contrário, mas, do mesmo modo, com igual importância, fadiga nãoexplicada, confusão, cansaço, edema, náuseas e vômitos, diaforese, arritmias, tosse ou dispnéia devem alertar o clínico para a possibilidade de infarto do miocárdio silencioso (18).

Quanto à mortalidade relacionada à neuropatia autonômica, em estudo de O'Brien e cols., fica ilustrada a importância da NAC. Os autores compararam a importância de vários fatores associados com mortalidade e observaram que a neuropatia autonômica foi um fator preditivo independente mais importante do que PAS, pé diabético, índice de massa corporal (IMC), neuropatia sensitiva, proteinúria e doença macrovascular (51).

Também foi observado que um aumento da mortalidade cardiovascular, assim como a mortalidade por todas as causas, estava associado com NAC no Hoorn Study (51). Utilizando-se 11C-HE e 123I MIBG como marcadores de neurotransmissão, tem sido observada desnervação cardíaca distal relacionada ao diabetes tanto em humanos $(48,49)$ como em ratos (2). Em humanos, além disso, um estudo mostrou que áreas de hiperatividade simpática estão presentes no miocárdio proximal de indivíduos com NAD grave. Acredita-se que a presença dessas áreas aumente o risco de arritmias cardíacas e morte súbita durante a ocorrência de isquemia miocárdica, o que seria uma explicação para a especial cardioproteção obtida com o uso de beta-bloqueadores nesses indivíduos. Em pacientes com DMl, disfunção simpática foi freqüente quando apresentavam microangiopatia, caracterizando-se por aumento do tônus e da resposta simpática e por perfusão miocárdica comprometida, os quais poderiam contribuir para as lesões do miocárdio que ocorrem no diabetes $(5)$.

O mecanismo pelo qual a NAC aumenta a mortalidade permanece incerto, podendo-se pressupor várias possibilidades (tabela 2 ) (51). Parte desta associação pode ser decorrente da alta prevalência de outras complicações e fatores de risco em pacientes diabéticos com neuropatia diabética autonômica cardiovascular. Por exemplo, a progressão de complicações microvasculares, tais como a nefropatia diabética, parece ser paralela à neuropatia autonômica cardiovascular e
Tabela 2. Possíveis mecanismos pelos quais a neuropatia autonômica cardiovascular pode aumentar a morbidade e mortalidade cardiovascular no diabetes mellitus.

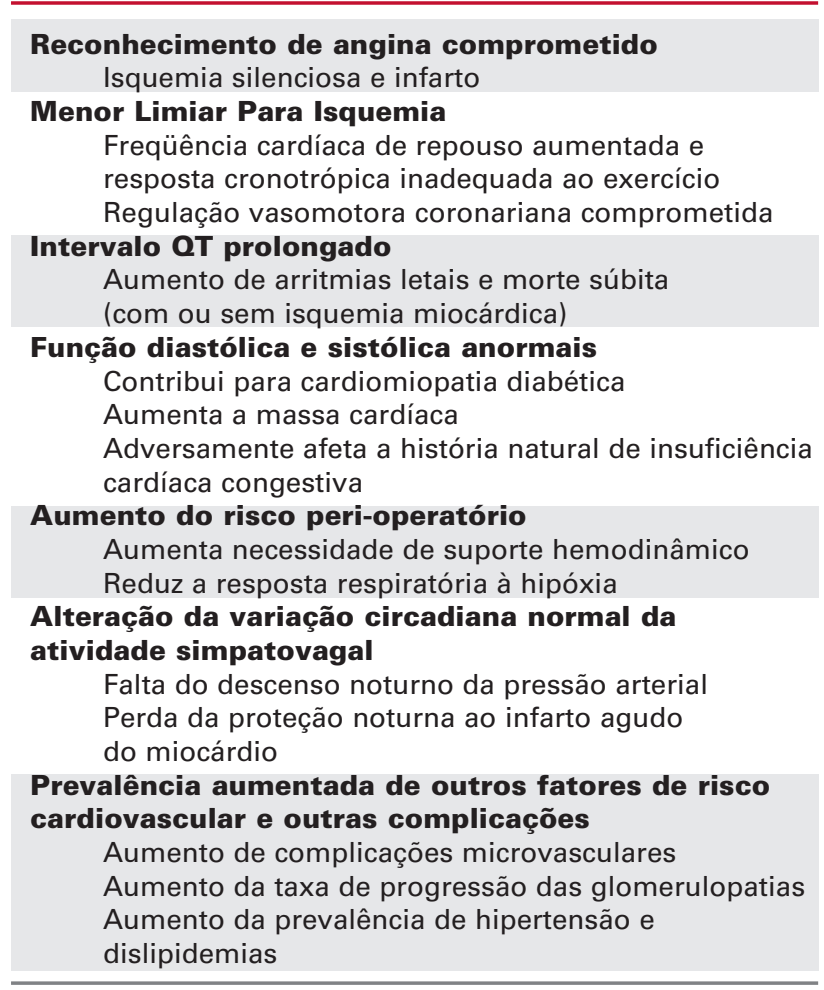

pode resultar em um aumento do risco cardiovascular relacionado à hipertensão e à dislipidemia. A NAC pode também diminuir a percepção à isquemia miocárdica. Há muito que clínicos descrevem que pacientes diabéticos apresentam poucos sintomas anginosos quando comparados com não-diabéticos. Assim, comparados com indivíduos não-diabéticos, com exercício limitado por isquemia, indivíduos com NAC menos provavelmente são limitados pela ocorrência de angina quando apresentam depressão do segmento ST. A taquicardia de repouso, uma manifestação inicial da desnervação parassimpática da NAC, aumenta a demanda miocárdica de oxigênio e pode colocar o paciente diabético mais próximo do limiar isquêmico. Além disso, a NAC pode alterar a regulação do fluxo coronariano, determinando resposta vasoconstritora a estímulos que em condições normais são vasodilatadores. Assim, a NAC poderia determinar episódios isquêmicos mais graves por alterar o balanço entre o suprimento miocárdico e a demanda (51). Além disso, a desnervação simpática cardíaca distal (figura 3), associando-se com áreas que apresentam inervação normal ou que estejam até hiperinervadas, favorece um desbalanço elétrico propício à ocorrência de arritmias. Por outro lado, observamos que, no $\mathrm{DMl}$, a disfunção 
simpática se associa com menor reserva de fluxo miocárdico e disfunção diastólica (5).

Intervalos QT prolongados em pacientes diabéticos correlacionam-se com o grau de neuropatia autonômica e podem predispor a sérias arritmias e morte súbita (51).

\section{PREVENÇÃO DE NAC}

Considerando-se a patogênese da neuropatia autonômica, pressupõe-se que a profilaxia de complicações com drogas que diminuem a velocidade de instalação das lesões em órgãos-alvo, através do controle metabólico, diminuição da pressão arterial e do controle dos lipídios séricos, como é o caso de medidas não-farmacológicas e farmacológicas, também determine diminuição das repercussões das lesões autonômicas no organismo. Exemplo é o uso da aspirina e dos inibidores da enzima conversora da angiotensina (IECA) (18).

\section{Manutenção de controle metabólico}

O estudo clássico de Pirart (52), que seguiu 4.400 diabéticos durante 25 anos, encontrou 12\% de neuropatia clinicamente detectável no início do estudo e 50\% ao fim de 25 anos de acompanhamento, estabelecendo historicamente a relação entre diabetes mellitus cronicamente descompensado e prevalência de ND. Tanto em pacientes com DMl (DCCT) como nos com DM2 [UK Prospective Diabetes Study (UKPDS)], foi demonstrada diminuição da velocidade de progressão da neuropatia associada ao controle metabólico (21,53-56). Embora a progressão da neuropatia autonômica não tenha sido muito bem definida nestes estudos, presume-se que os benefícios possam ser estendidos a ela.

\section{Antioxidantes}

Várias evidências sugerem que o estresse oxidativo aumentado, que ocorre nos vasos e nervos diabéticos, tenha importante papel na patogênese da neuropatia. Estudos têm mostrado que um potente antioxidante, o ácido alfa-lipóico, pode diminuir os sintomas e modificar a história natural da polineuropatia diabética, tanto no que se refere a testes de condução nervosa como autonômicos. Novos ensaios clínicos estão sendo realizados nos Estados Unidos e na Europa, avaliando sua eficácia (17).

O ácido alfa-lipóico parece diminuir a velocidade de progressão da neuropatia ou até revertê-la, porém novos estudos são necessários (18).

\section{Inibidores de aldose redutase}

Os inibidores de aldose redutase (57) reduzem o fluxo de glicose através da via dos polióis, inibindo o acúmulo de sorbitol e frutose no tecido e prevenindo processos oxidativos danosos à função do nervo. Alrestatin, sorbinil e tolrestatin são exemplos de inibidores de aldose redutase que foram utilizados em vários países, mas cujo uso foi abandonado devido à hepatotoxicidade e a outros efeitos colaterais importantes, como linfadenopatia, febre e pancitopenia. Atualmente, o único representante deste grupo que vem sendo utilizado é o epalrestat (Japão). Novos estudos utilizando agentes com baixa toxicidade deverão ser realizados.

\section{Ácido gama-linolênico}

O ácido gama-linolênico é um ácido graxo essencial metabolizado em ácido diomo-gama-linolênico, que constitui importante elemento fosfolipídico da membrana do neurônio. É um dos componentes do óleo primrose. É substrato para a formação de prostanóides, importantes para a manutenção da perfusão sangüínea do nervo. Em diabéticos, a reação de conversão inicial está inibida e, conseqüentemente, há diminuição na produção de seus metabólitos, o que pode contribuir para a patogênese da ND. Alguns estudos clínicos preliminares apresentaram resultados satisfatórios após um ano de uso (melhora na condução nervosa e nos déficits) $(58,59)$. Não há dados sobre disfunção autonômica.

\section{Inibidores da formação de produtos de glicação avançada}

A aminoguanidina é um dos inibidores que mostraram bom efeito terapêutico quando usada na nefropatia e na polineuropatia em diabetes experimental em ratos. Ainda não há comprovação clínica bem estabelecida do seu efeito na neuropatia diabética humana (17).

\section{Vasodilatadores}

Um estudo mostra que um IECA, o lisinopril, pode ter um efeito benéfico na evolução da ND somática. Embora os resultados possam ser promissores, estudos clínicos mais amplos ainda são necessários para se obter confirmação definitiva sobre essa nova oportunidade terapêutica (17). Além disso, o trandolapril reduz a progressão de infarto do miocárdio para insuficiência cardíaca grave em $62 \%(\mathrm{p}<0,001)$, efeito benéfico não visível em não-diabéticos, e que poderia estar relacionado com a evolução da neuropatia $(51)$. 


\section{Fatores de crescimento neurotróficos}

$\mathrm{O}$ uso de fatores de crescimento neurotróficos se encontra em fase experimental, e há projetos de terapia específica, como, por exemplo, o uso de fator de crescimento nervoso (NGF) no tratamento de neuropatia com predominância de acometimento de fibras finas (60), e o uso de fator de crescimento semelhante à insulina 1 (IGF-1) e IGF-2 no caso de fibras grossas. Poucos resultados clínicos com NGF foram promissores, de modo que o desenvolvimento de estudos clínicos para testá-lo foi suspenso.

\section{Inibidores da proteína quinase C}

Tem sido observado em vários estudos um aumento da glicose intracelular em neurônios, que favorece aumento dos níveis intracelulares de proteína quinase C, a qual, por sua vez, determina alterações na expressão da sintase do ácido nítrico endotelial e do fator de crescimento vascular endotelial (VEGF). Em estudos preliminares tem sido sugerido que um inibidor da proteína quinase $\mathrm{C}$, isoforma $\beta$, melhora medidas da função nervosa em pacientes com diabetes. Ensaios clínicos testando os efeitos desses inibidores encontram-se em andamento (17).

\section{TRATAMENTO DAS REPERCUSSÕES DA NAC}

O uso de beta-bloqueadores lipofílicos (propranolol) ou cardiosseletivos (atenolol) pode modular os efeitos da disfunção autonômica. Pelo fato de se oporem ao estímulo simpático, essas drogas podem restabelecer o equilíbrio simpático/parassimpático. Recentemente, o uso de metoprolol em pacientes com DMl tratados com ramipril e com albuminúria elevada mostrou diminuir a disfunção autonômica (18).

Conforme já citado, o trandolapril reduz a progressão de infarto do miocárdio para insuficiência cardíaca grave em $62 \%(\mathrm{p}<0,001)$, efeito benéfico não visível em não-diabéticos, e que poderia estar relacionado com a evolução da neuropatia (51). Por outro lado, no GISSI-3, o lisinopril reduziu em 6 semanas e 6 meses a mortalidade em um sub-grupo de pacientes diabéticos quando iniciado dentro de 24 horas após um infarto agudo do miocárdio.

Em relação ao efeito de beta-bloqueadores em pacientes diabéticos, as vantagens são indiscutíveis: determinam redução da mortalidade e re-infarto naqueles que sofreram um infarto miocárdico. Esses benefícios também ocorrem em diabéticos com insuficiência cardíaca de diferentes gravidades. Os benefícios do uso de beta-bloqueadores na insuficiência cardíaca, em diabéticos, têm sido observados com beta-bloqueadores não seletivos (isto é, bucindolol), com betabloqueadores cardio-seletivos (metoproplol) e com um beta-bloqueador não cardio-seletivo que também bloqueia receptores alfal adrenérgicos (carvedilol) (51). O benefício maior dos beta-bloqueadores nos pacientes diabéticos pode ser decorrente de vários fatores, entre eles a restauração de um balanço simpato-vagal em pacientes com neuropatia autonômica cardiovascular $(5,51)$. Apesar de inúmeras evidências de benefícios, infelizmente essas drogas ainda são pouco prescritas aos pacientes com diabetes (51).

Ao recomendar a realização de exercícios a pacientes com NAC, deve-se ter muito cuidado porque os pacientes freqüentemente têm baixa tolerância a eles e pelo fato de, com o exercício, haver possibilidade de precipitação de uma isquemia miocárdica sem dor (18).

$\mathrm{Na}$ realidade, tem sido demonstrado que o treinamento físico melhora a disfunção autonômica de pacientes com alterações leves. A realização de programa individualizado, de acordo com as respostas dos pacientes aos testes cardiovasculares autonômicos, e da avaliação da presença de cardiopatia isquêmica é necessária (18).

\section{REFERÊNCIAS}

1. Neumann C, Branchtein L, Schmid H. Severe autonomic neuropathy how many symptoms? Diabetes Care 1995; 18(1):133-4.

2. Schmid H, Forman L, Sherman P, Stevens M. Heterogeneous cardiac sympathetic denervation and decreased myocardial nerve growth factor in streptozotocin diabetic rats implications for cardiac sympathetic dysinnervation complicating diabetes. Diabetes 1999;48:603-8.

3. Neumann C, Schmid H. Relationship between the degree of cardiovascular autonomic dysfunction and symptoms of neuropathy and other complications of diabetes mellitus. Braz J Med Biol Res 1995;28:751-7.

4. Neumann C, Schmid H. Standardization of a computerized method for calculating autonomic function tests responses in healthy; subjects and patients with diabetes mellitus. Braz J Med Biol Res 1997;30:197-205.

5. Pop-Busui R, Kirkwood I, Schmid H, Marinescu V, Schroeder J, Larkin D, et al. Sympathetic dysfunction in type 1 diabetes: association with impaired myocardial blood flow reserve and diastolic dysfunction. J Am Coll Cardiol 2004;44(12):236874.

6. Schmid H, Schaan B, Cecconello F, Maestri T, Neumann C. Proliferative diabetic retinopathy is related to cardiovascular autonomic neuropathy in non-insulin-dependent diabetes mellitus. Diabetes Res Clin Pract 1995;29(3):163-8.

7. Neumann C, Martinez D, Schmid H. Nocturnal oxygen desaturation in diabetic patients with severe autonomic neuropathy. Diabetes Res Clin Pract 1995;28(2):97-102.

8. Schaan BD, Lacchini S, Bertoluci M, Irigoyen MC, Machado UF, Schmid H. Increased renal GLUT 1 abundance and urinary TGF $\beta 1$ in streptozotocin-induced diabetic rats implications for the development of nephropathy complicating diabetes. Horm Metab Res 2001;33:664-9. 
9. D'Agord Schaan B, Lacchini S, Bertoluci MC, Irigoyen MC, Machado UF, Schmid H. Impact of renal denervation on renal content of GLUT1, albuminuria and urinary TGF-beta1 in streptozotocin-induced diabetic rats. Auton Neurosci 2003;104(2):88-94.

10. Schaan BD, Irigoyen MC, Lacchini S, Moreira ED, Schmid H, Machado UF. Sympathetic modulation of the renal glucose transporter GLUT2 in diabetic rats. Auton Neurosci 2005; 117(1):54-61.

11. Schaan BD, Dall'Ago P, Maeda CY, Ferlin E, Fernandes TG, Schmid $\mathrm{H}$, et al. Relationship between cardiovascular dysfunction and hyperglycemia in streptozotocin-induced diabetes in rats. Braz J Med Biol Res 2004;37(12):1895-902.

12. Schaan BD, Maeda CY, Timm HB, Medeiros S, Moraes RS, Ferlin E, et al. Time course of changes in heart rate and blood pressure variability in rats with streptozotocin-induced diabetes treated with insulin. Braz J Med Biol Res 1997;30(9):1081-6.

13. Ferreira $A$. Uso da estesiometria e biotesiometria para o diagnóstico de polineuropatia periférica do diabete melito e do pé diabético em risco de ulceração neuropática. Dissertação (Pós-graduação em Medicina, Clínica Médica). Universidade Federal do Rio Grande do Sul, 1997.

14. American Diabetes Association, American Academy of Neurology Report and recommendations of the San Antonio Conference on Diabetic Neuropathy Consensus Statement. Diabetes Care 1988;11:592-7.

15. American Diabetes Association and American Academy of Neurology. Proceedings of a consensus development conference on standardized measures in diabetic neuropathy. Diabetes Care 1992;15:1080-107.

16. Thomas PK. Classification of the diabetic neuropathies. In: Gries FA, Cameron NE, Low PA, Ziegler D. Textbook of Diabetic Neuropathy. Georg Thieme Verlag, 2003.

17. Boulton AJ, Malik RA, Arezzo JC, Sosenko JM. Diabetic somatic neuropathies. Diabetes Care 2004;27:1458-86.

18. Vinik Al, Maser RE, Michell BD, Freeman R. Diabetic autonomic neuropathy. Diabetes Care 2003;26(5):1553-79.

19. Meyer C, Grossmann R, Mitrakou A, Mahler R, Veneman T, Gerich J, et al. Effects of autonomic neuropathy on counterregulation and awareness of hypoglycemia in type 1 diabetic patients. Diabetes Care 1988;21:1960-6.

20. Shaw JE, Zinmet PZ, Gries FA, Ziegler D. Epidemiology of diabetic neuropathy. In: Gries FA, Cameron NE, Low PA, Ziegler D. Textbook of Diabetic Neuropathy. Georg Thieme Verlag, 2003.

21. DCCT Research Group. The effect of intensive treatment of diabetes on the development and progression of long-term complications in insulin-dependent diabetes mellitus. N Engl J Med 1993;329:977-86.

22. Feldman EL, Stevens MJ, Greene DA. Pathogenesis of diabetic neuropathy. Clin Neurosci 1997;4(6):365-70.

23. Brownlee M. Glycation products and the pathogenesis of diabetic complications. Diabetes Care 1992;15:1835-43.

24. Greene DA, Stevens MJ, Obrosova I, Feldman EL. Glucoseinduced oxidative stress and programmed cell death in diabetic neuropathy. Eur J Pharmacol 1999;375(1-3):217-25.

25. Dyck PJ, Lais A, Karnes JL, O'Brien P, Rizza R. Fiber loss is primary and multifocal in sural nerves in diabetic polyneuropathy. Ann Neurol 1986;19(5):425-39.

26. Stevens MJ, Feldman EL, Greene DA. The aetiology of diabetic neuropathy the combined roles of metabolic and vascular defects. Diabet Med 1995;12(7):566-79.

27. Brownlee M. Biochemistry and molecular cell biology of diabetic complications. Nature 2001;414:813-20.

28. Feldman EL. Oxidative stress and diabetic neuropathy: a new understanding of an old problem. J Clin Invest 2003; 111:431-3.

29. Hellweg R, Hartung HD. Endogenous levels of growth factor are altered in experimental diabetes mellitus: a possible role of NGF on the pathogenesis of diabetes neuropathy. J Neurosci Res 1990;26:258-67.
30. Nickander KK, Schmelzer JD, Rohwer DA, Low PA. Effect of alpha-tocopherol deficiency on indices of oxidative stress in normal and diabetic peripheral nerve. J Neurol Sci 1994; 126(1):6-14.

31. Feldman EL, Stevens MJ, Greene DA. Pathogenesis of diabetic neuropathy. Clin Neurosci 1997;4(6):365-70.

32. Ewing DJ, Campbell IW, Burt AA, Clarke BF. Vascular reflexes in diabetic autonomic neuropathy. Lancet 1973;2(7842):1354-6.

33. Campbell LV, Kraegen EW, Lazarus L. Defective blood glucose counterregulation in diabetic is a selective form of autonomic neuropathy. Br Med J 1977;2:1527-9.

34. Martyn CN, Ewing DJ. Pupil cycle time: a simple way of measuring an autonomic reflex. J Neurol Neurosurg Psychiatry 1986;49(7):771-4.

35. Ewing DJ, Campbell IW, Clarke BF. Heart rate changes in diabetes mellitus. Lancet 1981;1(8213):183-6.

36. Ewing DJ, Campbell IW, Clarke BF. The natural history of diabetic autonomic neuropathy. $\mathbf{Q}$ J Med 1980;69:95-108.

37. Ewing DJ, Campbell IW, Clarke BF. Assessment of cardiovascular effects in diabetic autonomic neuropathy and prognostic implications. Ann Int Med 1980;92:308-11.

38. Spallone V, Maiello MR, Cicconetti E, Menzinger G. Autonomic neuropathy and cardiovascular risk factors in insulindependent and non insulin-dependent diabetes. Diabetes Res Clin Pract 1997;34(suppl. 3):169-79.

39. Ewing DJ, Borsey DQ, Bellavere F, Clarke BF. Cardiac autonomic neuropathy in diabetes: comparison of measures of R$R$ interval variation. Diabetologia 1981;21(1):18-24.

40. Kennedy WR, Navarro X, Sutherland DE. Neuropathy profile of diabetic patients in a pancreas transplantation program. Neurology 1995;45(4):773-80.

41. Ziegler D, Dannehl K, Muhlen H, Spuler M, Gries FA. Prevalence of cardiovascular autonomic dysfunction assessed by spectral analysis, vector analysis, and standard tests of heart rate variation and blood pressure responses at various stages of diabetic neuropathy. Diabet Med 1992;9(9):806-14.

42. Glowniak JV. Cardiac studies with metaiodobenzylguanidine: a critique of methods and interpretation of results. J Nucl Med 1995;36(11):2133-7. Review.

43. Mantysaari M, Kuikka J, Mustonen J, Tahvanainen K, Vanninen E, Lansimies E, et al. Noninvasive detection of cardiac sympathetic nervous dysfunction in diabetic patients using [123I] metaiodobenzylguanidine. Diabetes 1992;41(9):1069-75.

44. Langer A, Freeman MR, Josse RG, Armstrong PW. Metaiodobenzylguanidine imaging in diabetes mellitus assessment of cardiac sympathetic denervation and its relation to autonomic dysfunction and silent myocardial ischemia. J Am Coll Cardiol 1995;25:610-8.

45. Murata K, Araki S. Assessment of autonomic neurotoxicity in occupational and environmental health as determined by ECG R-R interval variability: a review. Am J Ind Med 1996;30(2):155-63.

46. Murata K, Sumida Y, Murashima S, Matsumura K, Takeda $H$, Nakagawa $T$, et al. A novel method for the assessment of autonomic neuropathy in type 2 diabetic patients: a comparative evaluation of 123I-MIBG myocardial scintigraphy and power spectral analysis of heart rate variability. Diabet Med 1996;13(3):266-72.

47. Rosenspire KC, Haka MS, Van Dort ME, Jewett DM, Gildersleeve DL, Schwaiger M, et al. Synthesis and preliminary evaluation of carbon-11-meta-hydroxyephedrine: a false transmitter agent for heart neuronal imaging. J Nucl Med 1990;31(8):1328-34.

48. Stevens MJ, Raffel DM, Allman KC, Dayanikli F, Ficaro E, Sandford T, et al. Cardiac sympathetic dysinnervation in diabetes implications for enhanced cardiovascular risk. Circulation 1998;98:961-8.

49. Stevens MJ, Raffel DM, Allman KC, Schwaiger M, Wieland DM. Regression of cardiac sympathetic dysinnervation complicating diabetes: an assessment by $\mathrm{C}-11$ hydroxyephedrine and positron emission tomography. Metabolism 1999; 48:92-101. 
50. O'Brien IA, McFadden JP, Corrall RJ. The influence of autonomic neuropathy on mortality in insulin-dependent diabetes. Q J Med 1991;79:495-502.

51. Nesto RW. Diabetes and heart disease. In: Zipes DP, Libby $P$, Bonow RO, Braunwald E. Heart Disease: A Textbook of Cardiovascular Medicine. $7^{\text {th }}$ ed. Philadelphia: WB Saunders Co., 2005. p. 1355-66.

52. Pirart J. Diabetes mellitus and its degenerative complications: a prospective study of 4,400 patients observed between 1947 and 1973. Diabetes Care 1978;1:168-88.

53. Amthor KF, Dahl-Jorgensen K, Berg TJ, Sandvik L, Hanssen KF. The effect of 8 years of strict glycaemic control on peripheral nerve function in IDDM patients: the Oslo study. Diabetologia 1994;37:579-86.

54. Ohkubo Y, Kishikawa H, Araki E, Miyata T, Isami S, Motoyoshi $S$, et al. Intensive insulin therapy prevents the progression of diabetic microvascular complications in Japanese patients with non-insulin-dependent diabetes: a randomized prospective 6-year study. Diabetes Res Clin Pract 1995;28:103-17.

55. Intensive blood-glucose control with sulphonylureas or insulin compared with conventional treatment and risk of complications in patients with type 2 diabetes (UKPDS 33). UK Prospective diabetes Study (UKPDS) Group. Lancet 1998;352(9131):837-53.
56. Kennedy WR, Navarro X, Goetz FC, Sutherland DE, Najarian JS. Effects of pancreas transplantation on diabetic neuropathy. N Engl J Med 1990;322:1031-7.

57. Boulton AJM. Current and emerging treatments for the diabetic neuropathies. Diabetes Rev 1999;7:279-386.

58. $\gamma$-Linolenic Acid Multicenter Trial Group. Treatment of diabetic neuropathy with [gamma]-linolenic acid. Diabetes Care 1993; $16: 8-15$.

59. Hounsom L, Horrobin DF, Tritschler H, Corder R, Tomlinson DR. A lipoic acid-gamma linolenic acid conjugate is effective against multiple indices of experimental diabetic neuropathy. Diabetologia 1998;41:839-43.

60. Apfel SC. Nerve growth factor for the treatment of diabetic neuropathy: what went wrong, what went right, and what does the future hold? Int Rev Neurobiol 2002;50:393-413.

\section{Endereço para correspondência:}

Helena Schmid

Av. Sarmento Leite 245

90050-170 Porto Alegre, RS

Fax: (51) 3214-8246

E-mail: hschmid@fffcmpa.edu.br 